\title{
Double Strand Brachytherapy
}

National Cancer Institute

\section{Source}

National Cancer Institute. Double Strand Brachytherapy. NCI Thesaurus. Code C106073.

An internal radiation treatment technique in which radioactive seeds are embedded in suture material and directly affixed to target cancerous tissue. 